\title{
醛肜类神经性毒剂防治药物的新型解毒分子 作用机制
}

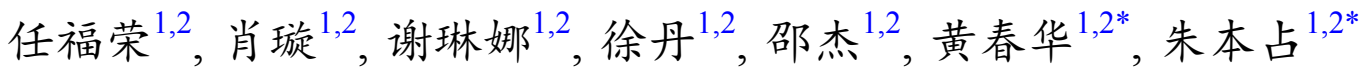

1. 中国科学院生态环境研究中心, 环境化学与生态毒理学国家重点实验室, 北京 100085

2. 中国科学院大学, 北京 100049

*通讯作者, E-mail: bjsyhch@126.com; bzhu@rcees.ac.cn

收稿日期：2018-04-07; 接受日期：2018-08-08; 网络版发表日期: 2018-09-21

中国科学院战略性先导科技专项(B类) (编号: XDB01020300)和国家自然科学基金(编号: 21577149, 21477139, 21621064, 21777180)资助项目

摘要吡啶盐醛肟类药物是一类常见的有机磷类神经性毒剂和农药的解毒剂. 卤代苯醌是一类致癌性的活性 中间体，最近在饮用水消毒副产物中也被检出. 我们发现，2-吡啶盐醛肟类代表性药物如解磷定可快速促进四氯1,4-苯醌通过连续两步的脱氯水解反应转化为 2,5 -二氯-3,6-二着基-1,4-苯醌，比其自然水解的速率提高了18万倍. 然而，当解磷定中的氮羟基被掩蔽后则不能促进四氯-1,4-苯醌水解。通过联合运用多种分离和分析鉴定方法发 现, 解磷定最终转化为其相应的腈类化合物. 采用 ${ }^{18} \mathrm{O}-\mathrm{H}_{2} \mathrm{O}$ 标记等实验, 最终确定2-吡啶盐醛肜类药物对四卤苯醌 类致癌物的解毒机理是通过亲核取代同时伴随两次Beckmann裂解反应。本文介绍了这类可在正常生理条件下发 生的连续两步Beckmann裂解反应, 以期对将来研究这两类具有重要生物学和环境学意义的醛肜类药物及多卤代 醌类环境致癌物具有指导作用。

关键词 Beckmann裂解反应, 吡啶盐醛肟, 神经性毒剂防治药物, 四氯-1,4-苯醌, 解毒机理

\section{1 引言}

有机磷(OP)农药和神经毒剂是经典的胆碱酯酶抑 制剂, 会造成乙酰胆碱累积从而导致类胆碱综合征. 严 重时会导致呼吸衰竭从而中毒致死. 常见的治疗有机 磷中毒的药物包括：阿托品(Atropine，抗毒䓬碱药 物)、安定(Diazepam) 和作为有机磷乙酰胆碱酯酶 (AChE)活化剂的四级 $N$-烷基吡啶醛肟类药物，即吡啶 盐醛肜类药物 ${ }^{[1 \sim 13]}$.

1951年，Wilson报道了羟胺可恢复OP抑制的
$\mathrm{AChE}$ 活性，即在使用1.2 $\mathrm{M} \mathrm{NH}_{2} \mathrm{OH}$ 处理 $1 \mathrm{~h}$ 之后，50\% $\mathrm{AChE}$ 的活性基本恢复 ${ }^{[2]}$. 进一步研究发现, $\mathrm{AChE}$ 的活 性中心存在阴离子位点(该位点在磷酸化之后仍然保 持完好), 因此他们考虑可以设计一种用于 $\mathrm{OP}$ 解毒的 定点的肜类解毒剂, 即碘化 $N$-甲基-2-吡啶醛肜(2$\mathrm{PAM}$ ). 基于这样的结构，基团肜可以增强分子的亲核 性 $(\alpha$-亲核试剂), 而四级氮则使该分子可以与 $\mathrm{AChE}$ 的 阴离子部位很好地结合，同时使肜阴离子正好朝向磷 原子, 这种合适的分子构造使得2-PAM对AChE的活化 十分高效, 因此之后被广泛用作解毒剂. 在 20 世纪 50 年

引用格式: Ren FR, Xiao X, Xie LN, Xu D, Shao J, Huang CH, Zhu BZ. A new detoxification mechanism for aldoxime therapeutic antidotes for chemical warfare nerve-agents . Sci Sin Chim, 2018, 48, doi: 10.1360/N032018-00084 
代，Wilson及其同事的这项先驱性工作对于此后 50 年 间超过 1000 种肟类活化剂的合成和评估无疑具有奠基 性意义，促使众多研究者致力于该类物质的研究. 2PAM和几种双四级吡啶醛肜(图1)在某些国家已用于 临床治疗. 在众多神经毒剂抑制的人类AChE的解毒剂 中, Hlo-7目前被认为是最经典的体外活化剂, 是一种 非常有优势的醛肜类药物.

在对OPs进行亲核取代反应中, $\alpha$-亲核试剂 $\mathrm{Pyr}^{+}-$ $\mathrm{CH}=\mathrm{N}-\mathrm{O}^{-}$对磷原子进攻. 实际上，肟阴离子的浓度取 决于其酸度常数 $\mathrm{p} K_{\mathrm{a}}$ 和溶剂的 $\mathrm{pH}, \mathrm{p} K_{\mathrm{a}}$ 值的大小表现出 肜阴离子对亲电试剂(如 $\mathrm{OP}$ )的亲和力. 吡啶盐醛肜类 药物的 $\mathrm{p} K_{\mathrm{a}}$ 一般为7.3 8.5 (2-PAM: 7.68; HI-6: 7.28; TMB-4: 7.78 8.2; Obidoxime: 7.54 8.48) ${ }^{[14 \sim 20]}$. 值得 一提的是, 2-PAM较3-PAM和4-PAM对AChE的活化效 果要好, 这可能与后面两种醛肟的 $\mathrm{p} K_{\mathrm{a}}$ 较大 $(3-\mathrm{PAM}$ : $\mathrm{p} K_{\mathrm{a}}>9$; 4-PAM: $\mathrm{p} K_{\mathrm{a}}=8.27$ )以及醛肜基团的位置(影响 对 $\mathrm{P}$ 原子的进攻)有关 ${ }^{[21 ~ 23]}$.

如图2所示, 酫肜在活化AChE后, 最终生成磷酸化 的肜(phosphylated oximes, POXs), 这是一种经典的中 间体，其产生浓度很低并且不稳定，会减慢活化的过 程. 因此，在寻找新的肜类活化剂时，必须考虑到 POXs的性质, 评估它在体外的动力学过程. 在生理 $\mathrm{pH}$ 条件下, 醛弜POXs的稳定性要低于酮肜; 2-吡啶醛肪 的POXs 的水解半衰期 $t_{1 / 2}$ 约为 $1 \mathrm{~min}$, 较 4 -吡啶醛肜
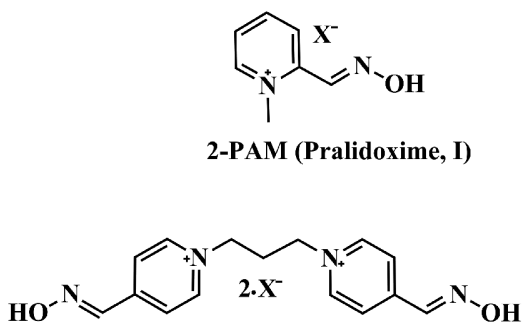

TMB-4 ( Trimedoxime, III)

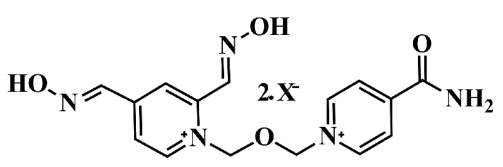

HLo-7, V
POXs的水解速率快50 100倍. 基于以上讨论，醛肜基 团处于四级氮邻位的 3 种醛肪 $(I 、 I I 、 V)$ 形成的POXs 比处于对位的3种醛肜(III、IV、VI) 有优势, 因为前者 分解速率快，不会阻碍OP-AChE的再生成. 值得一提 的是, POXs 是 $\mathrm{OP}$ 水解酶(如细菌磷酸三酯酶、哺乳动 物对氧磷酶)很好的底物, 因此它们在体内的积累并不 会达到影响酶活化的水平.

关于几种吡啶盐醛肜类药物的毒性, 对 $\mathbf{I} \sim \mathbf{V} 5$ 种醛 肜有大量的报道，大部分认为其毒性与吡啶环上的四 级氮有关, 而不是醛肜基团. 在啮齿动物中, 醛肜 $I V$ 静 脉注射的半数致死量 $\mathrm{LD}_{50}$ 值为 $200 \sim 400 \mathrm{mg} / \mathrm{kg}$, 醛肟 IV 的毒性是I、II、V的3 6倍. 醛肜 VI是报道中毒性最低 的药物, $\mathrm{LD}_{50}$ 值为 $2950 \mathrm{mg} / \mathrm{kg}$, 而酫肜 III是这6种醛肜 中毒性最高的双四级氮吡啶醛肟. 有研究者定量评估 了醛胴 II、IV、V, 他们对肟在实验动物狗和猴子中 导致的毒性症状进行了记录和排序 ${ }^{[2]}$. 他们评估醛肜 II、IV、V影响 10\%猴子的计量分别为616、133和 $296 \mu \mathrm{mol} / \mathrm{kg}$, 这与啮齿动物的毒性测定结果一致. 在 接近致死剂量时, 最常见的中毒症状为呕吐、分泌唾 液、反应迟缓和抽搐. 在人体中, 也进行了吡啶盐醛 肟类药物的安全性测试. 选择750位志愿者, 静脉注射 肜类药物250 500 mg后，不良反应主要有血压变化、 脉搏变化、头晕、恶心、视力模糊; 经口摄入醛朊的 主要不良反应是肠胃不适.

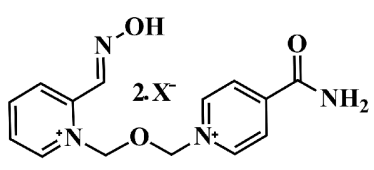

HI-6, II

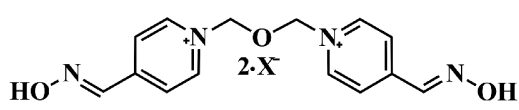

Obidoxime, IV<smiles></smiles>

MMB-4 ( Methoxime, VI)

图 1 几种吡啶盐醛肜类活化剂的化学结构式 ${ }^{[2]}$

Figure 1 Chemical structures of several pyridinium aldoxime reactivators [2]. 


\section{$\operatorname{AChE}$ 的活化过程：}

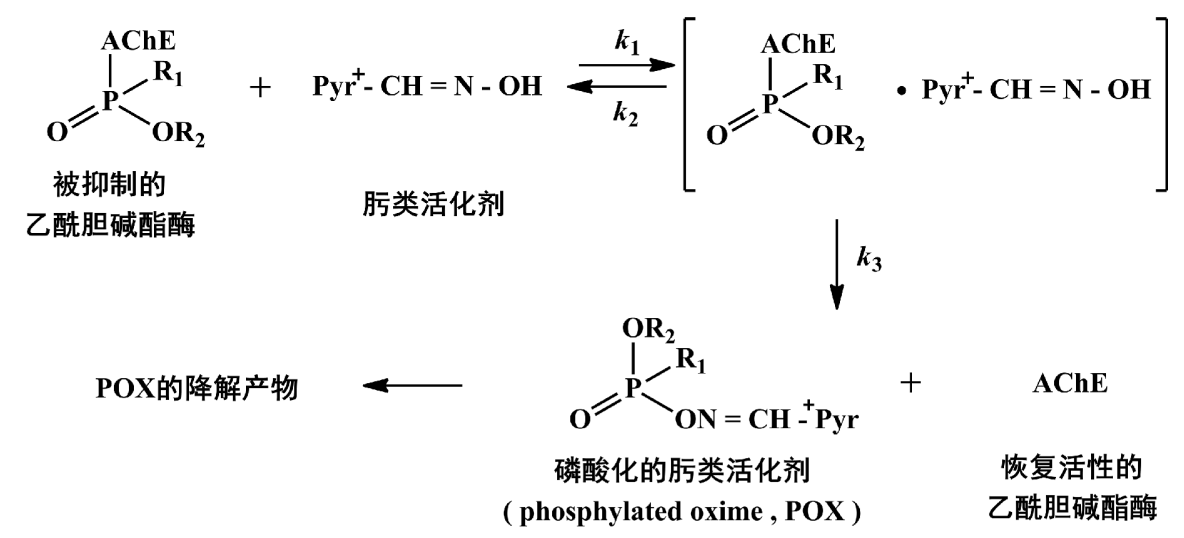

图 2 肟类药物活化磷酸化乙酰胆碱酯酶的反应 ${ }^{[2]}$

Figure 2 Proposed scheme of chemical reactions involved in the reactivation of phosphorylated cholinesterase by oxime reactivator [2].

五氯酚(pentachlorophenol，PCP)是一种在世界范 围内广泛存在的持久性环境污染物, 它的存在给环境 和人类健康安全带来巨大威胁 ${ }^{[24]}$. 2009 年, PCP及其盐 和酯类被列入斯德哥尔摩公约，成为了持久性有机污 染物(POPs)的一员. 已经有充足的流行病学数据证明, PCP的暴露可以导致非霍奇金淋巴癌(Non-Hodgkin lymphoma), 因此2016年国际癌症研究组织(IARC)已 经将PCP的致癌等级从Group 2B (人类可能致癌物)提 升至Group 1 (人类致癌物) ${ }^{[25 ~ 29]}$. 此外, 近年来研究证 明, 在PCP的生产与应用中, 会产生含有高毒性的氯代 二苯并二恶英(PCCDs)、四氯代二苯并呋喃(TCBDFs) 等多种污染物 ${ }^{[30]}$.

$\mathrm{PCP}$ 是一种氯代的酚类化合物，主要被用作木材 保护剂、杀虫剂、除草剂 ${ }^{[31]}$. 在中国和其他发展中国 家, PCP还被用于杀灭钉螺以预防血吸虫病. 从 20 世纪 50 年代中期开始, 血吸虫病在中国长江流域的多省流 行. 其中 12 个省受到了血吸虫病的影响, 有大约 1200 万人感染, 几百万人死亡. $\mathrm{PCP}$ 在当年控制血吸虫病的 传播中发挥了巨大的作用. 但是由于 $\mathrm{PCP}$ 会危及人类 健康, 因此从1997年开始, 中国限制PCP及其钠盐的生 产. 然而, 由于血吸虫病在传统地区的再度流行, PCP 的生产再一次增加，在2003年的年产量高达3000 t. PCP的再次使用无疑对环境和人类健康带来了影响. 因为应用广泛和稳定性好, PCP成为无处不在的环境 污染物. 目前我国已将PCP列为高毒农药禁止生产和 使用，但是 $\mathrm{PCP}$ 造成的环境残留污染以及其环境毒性
效应仍存在 ${ }^{[24]}$. 目前, 尽管PCP的毒性机制并不十分清 楚, 但是已经发现其醌类和半醌代谢产物在其中起着 重要的作用, 如图3所示.

在人体、大鼠细胞以及啮齿类动物的活体实验中 均发现, PCP可以被肝脏微酶体中的细胞色素P450s氧 化酶氧化脱氯形成四氯-1,4-氢醌(TCHQ). 生成的四氯 氢醌会继续被氧化，通过四氯半醌自由基进一步氧化 为四氯-1,4-苯醌( TCBQ). TCHQ和TCBQ 具有较强的 基因毒性和致癌性 ${ }^{[32 \sim 34]}$, 在氧化还原的过程中会产生 大量的活性氧, 进而发生氧化应激反应, 对生物大分 子造成损伤. 在各种活性氧中, 普遍认为是羟基自由 基 $(\cdot \mathrm{OH})$ 导致了DNA的氧化损伤. 那么羟基自由基是 如何产生的呢? 根据文献报道, 羟基自由基很可能是 在超氧阴离子自由基 $\left(\mathrm{O}_{2}{ }^{-{ }^{-}}\right)$转化为 $\mathrm{H}_{2} \mathrm{O}_{2}$ 之后, 再进一 步与金属离子反应产生的 ${ }^{[33,35,36]}$.

虽然PCP对DNA没有明显的毒性反应活性，但是 其代谢产物TCHQ在体外DNA、多种细胞系和小鼠的 肝脏中均可以导致DNA断裂. TCHQ还可以在V79细 胞的HPRT中心造成微核的形成以及变异, 在V79细胞 和小鼠中可以导致8-羟基-2-脱氧鸟苷 $(8-\mathrm{OH}-\mathrm{dG})$ 的形 成. 此外, TCHQ在肿瘤细胞中也可以诱导形成加合物 和缺嘌呤/缺嘧啶的位点(AP位点) $)^{[37-44]}$.

我们先前的研究发现, 氯代苯醌可以和 $\mathrm{H}_{2} \mathrm{O}_{2}$ 通过 不依赖于金属离子的途径产生高活性的羟基自由基 $(\cdot \mathrm{OH})^{[45]}$. 反应机制如图4所示: $\mathrm{H}_{2} \mathrm{O}_{2}$ 对TCBQ进行亲核 攻击, 形成不稳定的三氯氢过氧基-1,4-苯醌( TrCBQ- 


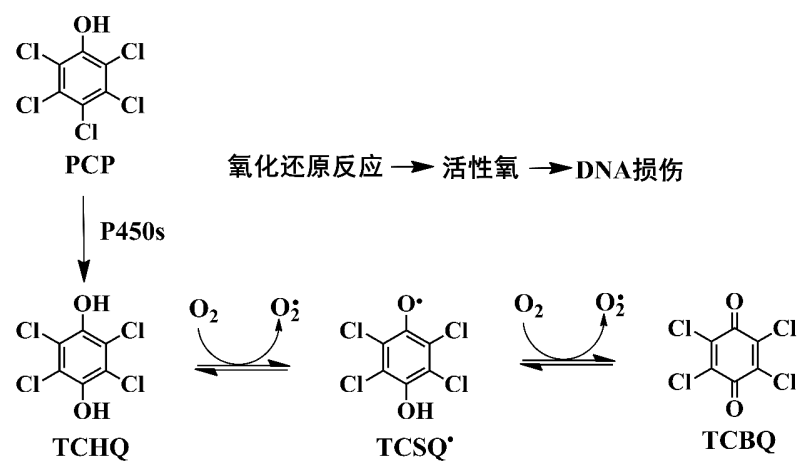

图 3 五氯酚(PCP)及其致癌性醌类和半醌类代谢物

Figure $3 \mathrm{PCP}$ and its carcinogenic quinone and semiquinone metabolite.

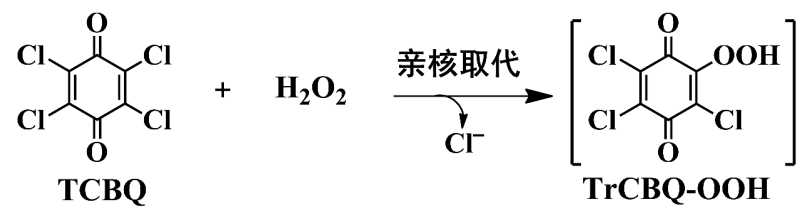

均裂

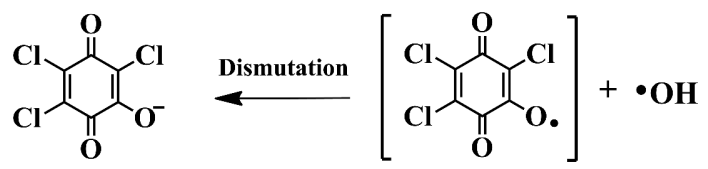

TrCBQ-O-

TrCBQ-O'

图 $4 \mathrm{TCBQ}$ 和 $\mathrm{H}_{2} \mathrm{O}_{2}$ 反应产生・ $\mathrm{OH}$ 的可能作用机制 ${ }^{[45]}$

Figure 4 Proposed mechanism for $\cdot \mathrm{OH}$ production by TCBQ and $\mathrm{H}_{2} \mathrm{O}_{2}[45]$.

$\mathrm{OOH}$ )中间产物，该中间产物均裂产生・OH和三氯-羟 基-1,4-苯醌自由基(TrCBQ-O•). TrCBQ-O•随后歧化形
成三氯-羟基-1,4-苯醌 (TrCBQ- $\left.\mathrm{O}^{-}\right)$. 有资料表明，卤代 醌与 $\mathrm{H}_{2} \mathrm{O}_{2}$ 产生・ $\mathrm{OH}$ 的反应可能在生物系统中存在，因 此这极可能是卤代醌具有毒性的重要原因之一.

2013年，Li课题组 ${ }^{[46-53]}$ 在饮用水消毒副产物中检 出了12种卤代醌(图5)，其中包括 TCBQ在内的其他低 氯代苯醌、澳代苯醌、碘代苯醌，这是除PCP等卤代 芳烃发生化学或生物代谢生成的途径以外, TCBQ类 卤代醌生成的又一途径. 对饮用水进行消毒是自 20 世 纪以来最重要的保护公共健康安全的举措之一, 它在 降低霍乱、伤寒和症疾传播等方面发挥了重要的作 用，保障了世界各国众多人口的饮用水安全. 然而，饮 用水消毒副产物(disinfection byproducts, DBPs)也随之 出现, 带来了新的健康问题, 据报道, 癌症(尤其是膀胱 癌)的发生和发展也可能与之相关. 消毒剂(如氯气和 氯胺)与水体中的天然有机质(natural organic matter, $\mathrm{NOM})$ 反应，可以产生DBPs. 从20世纪70年代以来，已 经检出了数百种DBPs, 然而, 从总有机氯含量分析, 这 些消毒副产物只占氯代消毒副产物的 $30 \%{ }^{[54,55]}$. 因此 对这些新型卤代醌类消毒副产物进行解毒研究十分 关键.

我们在研究羟胺类药物对有机农药及醌类致癌物 的解毒作用机制 ${ }^{[5611}$ 时意外发现，苯基异羟肜酸 (BHA) 可以对 TCBQ通过两次Lossen重排反应进行解 毒(图6) ${ }^{[58]}$. 首先, 一分子的BHA与 $\mathrm{TCBQ}$ 发生亲核取代 反应，形成中间体，中间体脱去氢质子，经过第一次 Lossen重排产生三氯一羟基-1,4-苯醌(TrCBQ-OH)和 一分子的苯基异㲵酸酯; 接着，另一分子的BHA与生 成的 $\operatorname{TrCBQ}-\mathrm{OH}$ 发生第二步亲核取代反应，并经过类<smiles>O=C1C=C(Cl)C(=O)C=C1Cl</smiles>

2,6-二氯苯醌 (2,6-DCBQ)<smiles>O=C1C=C(Br)C(=O)C(Br)=C1</smiles>

2,6-二溴苯醌 (2,6-DBBQ)<smiles>O=C1C=CC(Cl)C(Cl)=C1</smiles>

2,5-二氯苯醌 (2,5-DCBQ)<smiles>O=C1C=C(Br)C(=O)C=C1Br</smiles>

2,5-二溴苯醌 (2,5-DBBQ)

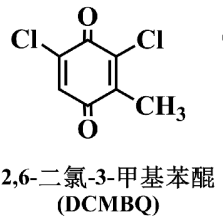<smiles>O=C1C=C(Cl)C(Cl)=CC1=O</smiles>

三氯苯醌
(TrCBO)<smiles>O=C1C(Br)=C(Br)C(=O)C(Br)=C1Br</smiles>

四溴苯醌 (TBBQ)

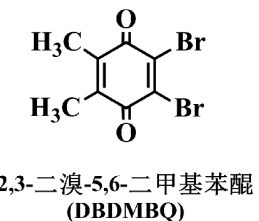
(DBDMBQ)<smiles>O=C1C(Cl)=C(Cl)C(=O)C(Cl)C1Cl</smiles>
四氯苯醌
(TCBQ)<smiles>O=C1C(Br)=C(Br)C(Br)=C1Br</smiles>

四溴邻苯醌 (O-TBBQ)<smiles>O=C1OC(=O)C(Cl)C(Cl)=C1Cl</smiles>

四氯邻苯醌 (O-TCBQ)<smiles>O=C1C=CC(=O)C(I)=C1I</smiles>

2,3-二碘苯醌 (DIBQ)

图 5 饮用水消毒副产物中检出的 12 种卤代醌 ${ }^{[46]}$

Figure 5 The structure of twelve halogenated quinones identified as chlorination disinfection byproducts in drinking water [46]. 


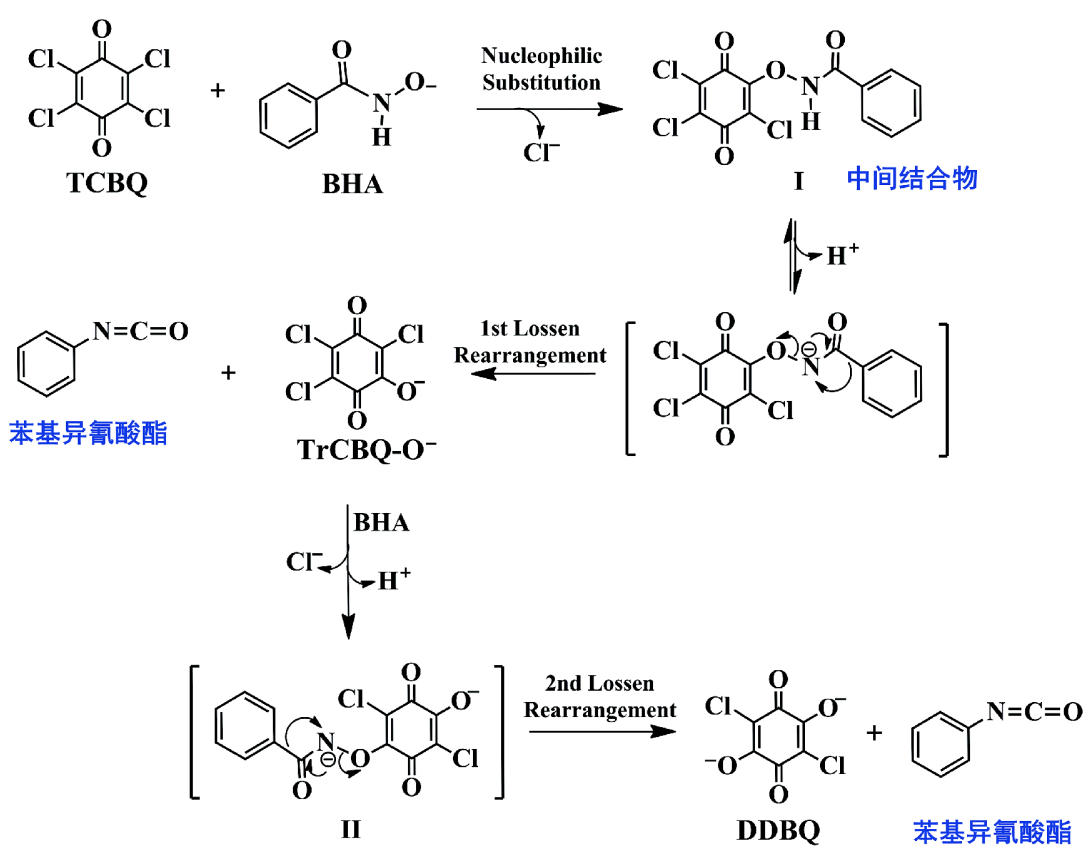

图 6 苯基异羟弜酸对四氯苯醌解毒的反应机制: 自杀式亲核进攻伴随着两次Lossen 重排反应 ${ }^{[58]}$

Figure 6 Proposed mechanism for the dramatic acceleration of TCBQ hydrolysis by BHA: suicidal nucleophilic attack coupled with an unusual double Lossen rearrangement [58].

似的中间体, 发生第二次Lossen重排反应, 最终生成二 氯二羟基-1,4-苯醌(DDBQ)和另一分子苯基异氧酸酯. 上述反应过程有两点优势: (1) 与原始反应物 TCBQ相 比, 终产物 $\mathrm{DDBQ}$ 毒性低得多, BHA促使 $\mathrm{TCBQ}$ 水解为 $\mathrm{DDBQ}$ 的速率与 $\mathrm{TCBQ}$ 自然水解为DDBQ的速率相比, 提高了 15 万倍; (2) 经典的Lossen重排需要在碱性或加 热条件下才能进行; 而TCBQ诱导的BHA重排反应可 以在室温条件下的中性甚至弱酸性环境中进行.

然而, 当把 $T C B Q$ 换为其他低氯代苯醌 $\left(\mathrm{C}_{n} \mathrm{BQ}\right)$ 时 发现, 它们与BHA的反应有所不同. 根据呈现出的反 应规律的不同, 我们将氯代苯醌分为两类: $\mathrm{DCBQ}$ 类和 $\mathrm{TCBQ}$ 类(图7). BHA与 $\mathrm{DCBQ}$ 类氯代苯醌反应时会生 成稳定的中间体，而与 $\mathrm{TCBQ}$ 类氯代苯醌则反应过快， 无法检测到中间体. 此外, 与 $\mathrm{DCBQ}$ 类反应生成的脱氯 水解产物的 $\mathrm{p} K_{\mathrm{a}}>2.5$, 而与 $\mathrm{TCBQ}$ 类反应生成的脱氯水 解产物的 $\mathrm{p} K_{\mathrm{a}} \leq 2.5^{[59]}$. 这说明BHA与氯代苯醌的反应 状况与氯代醌的氯原子取代数目、取代位置以及对应 醌的脱氯水解产物的 $\mathrm{p} K_{\mathrm{a}}$ 有关.

那么当将BHA替换为其结构类似物时, 又会出现 何种结果呢? 我们发现, BHA结构中氮原子上取代基 的微小改变, 或者氯代苯醌中氯原子数目发生变化, 都

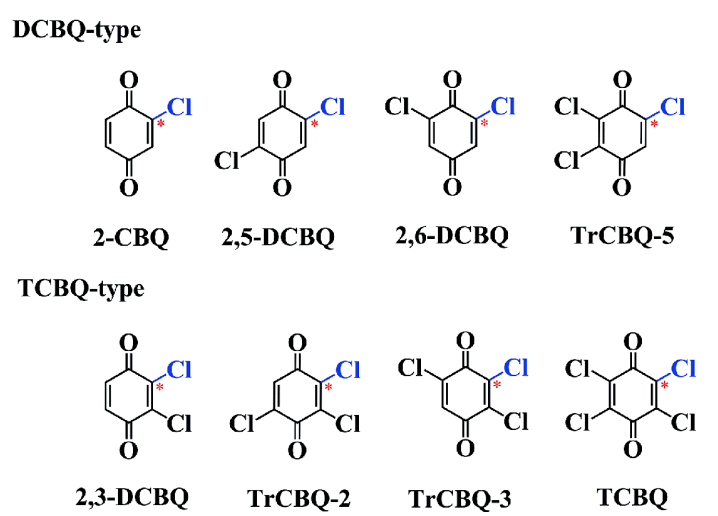

图 $7 \mathrm{DCBQ}$ 类和 $\mathrm{TCBQ}$ 类氯代苯醌. *代表反应位点 ${ }^{[59]}($ 网 络版彩图)

Figure 7 DCBQ-type and TCBQ-type chlorinated benzoquinones $(1: 1, *$ represents reaction site) [59] (color online).

会对反应机理有较大的影响. 当BHA结构中氮原子上 的氢变为甲基时, 即 $N$-MeBHA, 我们发现它同样可以 与 2,5 -二氯-1,4-苯醌发生亲核取代反应, 不同的是, 生 成的中间体的 $\mathrm{N}-\mathrm{O}$ 键发生均裂，生成氮自由基和醌氧 自由基, 醌氧自由基发生共振互变, 转化为醌酮自由 基，后者随后与氮自由基发生自由基偶联生成 $\mathrm{C}-\mathrm{N}$ 相 连的加合物, 该加合物同样可以发生烯醇-酮式共振互 
变, 生成最终产物. 当把BHA结构中N原子上的氢变为 苯基时，我们发现 $N$-苯基-BHA ( $N$-PhBHA) 同样会与 2,5-二氯-1,4-苯醌发生亲核取代反应，生成中间体 CBQ-O- $N$-PhBHA. 中间体随后发生类Claisen重排反 应, 即 $\mathrm{N}-\mathrm{O}$ 结合转变为 $\mathrm{C}-\mathrm{C}$ 键结合, 再经过酮-烯醇互 变, 最终得到产物.

有趣的是，我们前面提到的醛肟类药物在结构上 与 $\mathrm{BHA}$ 相似, 均含有 $\mathrm{N}-\mathrm{OH}$ 结构, 即均是羟胺的衍生 物，也均是 $\alpha$-亲核试剂. 但是二者不同的地方在于: 醛 肜类药物是 $\mathrm{C}=\mathrm{N}$ 双键, 而 $\mathrm{BHA}$ 与氮原子相连的是羰基. 既然醛肜类药物与BHA既有结构相似之处，也有明显 的结构区别，那么醛肜与卤代苯醌反应时的机理又会 与BHA存在何种异同之处呢? 它与卤代苯醌反应的具 体分子机理具体为何呢?

为此，我们针对 $\mathrm{TCBQ}$ 和吡啶盐醛肜类药物之间 的反应进行了仔细研究, 采用高效液相色谱串联质谱 (HPLC/MS)、核磁共振(NMR), 并结合O-18同位素标 记的方法对反应产物进行了分离、纯化和鉴定. 最终 发现, 吡啶盐醛肟类药物对 $\mathrm{TCBQ}$ 的解毒反应是一类 非同寻常的在正常生理条件下发生的连续两次Beckmann裂解反应.

\section{2 醛肜类神经性毒剂防治药物的新型解毒 分子作用机制}

\section{1 吡啶盐醛肜类药物加速 $\mathrm{TCBQ}$ 水解生成 DDBQ 的产率-时间的分析比较}

在中性水溶液中, TCBQ会先水解生成初级羟基 化中间产物一一三氯-一羟基-1,4-苯醌( TrCBQ-OH), 随后进一步缓慢水解生成最终的二羟基化产物— 2,5-二氯-3,6-二羟基-1,4-苯醌(2,5-dichloro-3,6-dihydroxy-1,4-benzoquinone, DDBQ $)^{[62]}$. 我们发现, 吡啶盐 醛肜类药物能够显著加速 $\mathrm{TCBQ}$ 的水解, 这一加速水 解的现象可以通过配备有紫外检测器的 HPLC在 DDBQ出峰处波长 $332 \mathrm{~nm}$ 的峰面积来定量. 在4种2-吡 啶盐醛肜类药物中, 2-PAM对 TCBQ的水解作用最为 高效(图8). 又考虑到2-PAM是自1955年以来在临床上 应用最早和最广的药物, 而且它在 4 种吡啶醛肟药物 中结构最简单，因此我们选择2-PAM作为模型化合物 来进行进一步的机理研究.

之前的研究发现, TCBQ的一级水解产物和二级

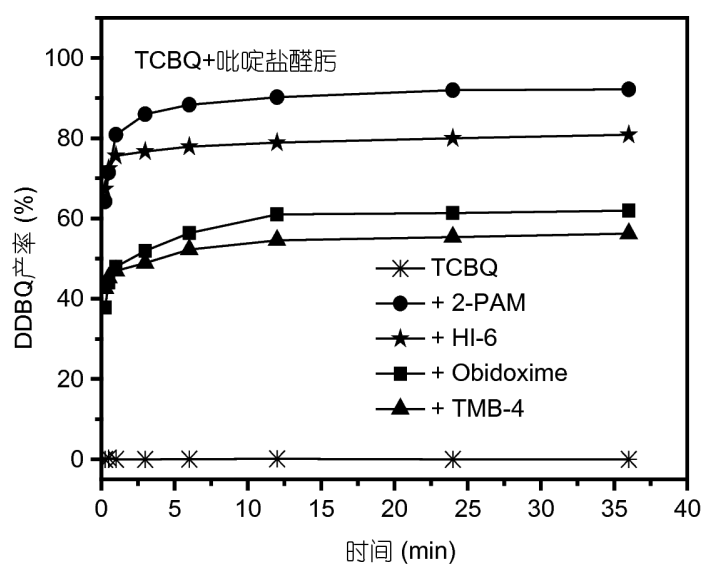

图 8 在2-PAM及其他吡啶盐醛肜类药物作用下, $\mathrm{TCBQ}$ 水 解产生DDBQ的产率-时间曲线 ${ }^{[61]}$

Figure 8 The yield-time curves for DDBQ by 2-PAM and other pyridinium aldoximes antidotes [61].

水解产物分别为 $T r C B Q-O H$ 和DDBQ, 通过电喷雾离 子化-四极杆-飞行时间-质谱(ESI-Q-TOF-MS)分析发 现, 在 TCBQ/2-PAM体系中, $\mathrm{TCBQ}$ 的脱氯水解过程也 是如此. 生成的 TrCBQ-OH和DDBQ的产量与反应物2PAM和 TCBQ 的摩尔比相关：低摩尔比(2-PAM: $\mathrm{TCBQ} \leq 1)$ 时，生成的产物主要为 $\mathrm{TrCBQ}-\mathrm{OH}$; 高摩尔 比(2-PAM:TCBQ $>1$ )时, TCBQ首先转变为 $T r C B Q-O H$, 随后进一步反应生成终产物DDBQ. 为证实2-PAM能 否直接加速 $\mathrm{TrCBQ}-\mathrm{OH}$ 水解转化为DDBQ, 我们根据 文献 $[58,62]$ 合成了 $\mathrm{TrCBQ}-\mathrm{OH}$, 结果发现确实如此.

值得注意的是，2-PAM促进四氯苯醌水解的反应 并不局限于两者之间，其他四卤代醌化合物(如四溴1,4 - 苯醌、四氟-1,4-苯醌、四氯- 1,4 -氢醌以及四氯1,2-苯醌)与2-PAM反应时, 都会发生类似的反应. 此 外,当把 $2-P A M$ 换为其他醛肟类药物, 如Trimedoxime、Obidoxime和HI-6时，也会对 TCBQ水解有类似 的促进作用. 这些研究结果表明, 醛肟类药物促使四 卤代苯醌水解的反应具有普遍性.

此外还发现，2-PAM促进TCBQ水解的速率也依 赖于二者的摩尔比, 摩尔比越高, 水解速率越快. 例如, 在 $0.2 、 0.5 、 1.0 、 2.0$ 和 $5.0 \mathrm{mM}$ 的 $2-\mathrm{PAM}$ 存在条件下 $(\mathrm{pH} 7.4), \mathrm{TCBQ}(0.05 \mathrm{mM})$ 水解成DDBQ的半衰期分别 为 $10 、 2.39 、 0.50 、 0.25$ 和 $<0.17 \mathrm{~min}$. 与之前文献报道 的TCBQ自然水解的半衰期 $21 \mathrm{~d}^{[62]}$ 相比, 2-PAM可以将 $\mathrm{TCBQ}$ 水解转化为 $\mathrm{DDBQ}$ 的速率提高 18 万倍 $(\mathrm{TCBQ}$, $0.05 \mathrm{mM}$; 2-PAM, $5.0 \mathrm{mM}$ ). 


\section{2 游离的 2-PAM阴离子在 $\mathrm{TCBQ}$ 加速水解过程 中的作用}

2-PAM介导的 $\mathrm{TCBQ}$ 水解转化为DDBQ的速率依 赖于缓冲液的 $\mathrm{pH}$. 当 $\mathrm{pH} \leq 4$ 时, $\mathrm{TCBQ}$ 几乎不发生水解, 但随着 $\mathrm{pH}$ 增加，水解速率逐步上升．这表明游离的2PAM阴离子可能是 2-PAM $\left(\mathrm{p} K_{\mathrm{a}}\right.$ 为7.68)参与反应的活 性态. 如果确实如此，那么游离的2-PAM阴离子被掩 蔽后, 应该不能促进 $\mathrm{TCBQ}$ 的水解. 为进一步证实此说 法是否属实，我们根据文献[63]合成了 $O$-甲基-2-PAM $\left(\mathrm{Py}^{+}\left(\mathrm{CH}_{3}\right)-\mathrm{CH}=\mathrm{N}-\mathrm{O}-\mathrm{CH}_{3}\right)^{[61]}$, 以阻止在反应中形成游 离的氧阴离子. 结果发现, $O$-甲基-2-PAM确实不能促 进 TCBQ的水解，即使当缓冲溶液的 $\mathrm{pH}$ 高达 10 时也无 任何促进作用. 上述结果明确表明，游离的2-PAM阴 离子对促进TCBQ水解至关重要.

\section{$2.32-P A M 与 T C B Q 反$ 应后的主要产物的分析鉴定}

当2-PAM存在时, TCBQ水解速率比其自然水解 的速率要快得多, 这似乎表明2-PAM可以催化这一水 解反应的进程. 然而我们发现, 在与 TCBQ反应的过程 中, 随着2-PAM的消耗, 伴随着生成了质荷比为 $m / z 119$ 的新物质. 这表明, 在 TCBQ的水解反应中, 2-PAM并不 是真正意义上的催化剂.

为了进一步探索 $\mathrm{TCBQ} / 2-\mathrm{PAM}$ 反应的分子机制,
我们联合运用HPLC-ESI-MS和NMR对2-PAM反应后 生成的终产物进行了分离、纯化、鉴定. 通过与标准 品比对, 发现产物为 $N$-甲基-2-吡啶甲腈(2-CMP) (反应 式(1)).

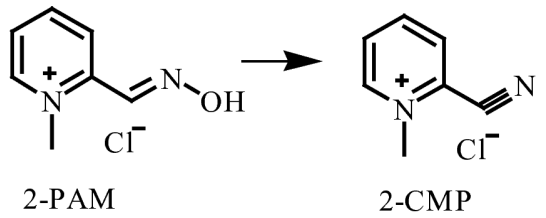

这说明, 2-CMP为2-PAM与 $\mathrm{TCBQ}$ 反应后生成的最 终产物, 其产率高达 $97.4 \%$. 不仅如此, 我们在 TrCBQ$\mathrm{OH} / 2$-PAM反应体系中也得到了类似的实验结果.

那么2-CMP是如何从2-PAM转化得到的呢? 根据 以往的文献报道，一个可能的典型反应路径是通过 Beckmann裂解反应，即将弜 $\left(\mathrm{R}_{1}-\left(\mathrm{R}_{2}\right) \mathrm{C}=\mathrm{N}-\mathrm{OH}\right)$ 经过氮 正离子或者碳正离子中间体转化为相应的腈 $\left(\mathrm{R}_{1}-\right.$ $\mathrm{C} \equiv \mathrm{N}$ )的反应 ${ }^{[2,64-67]}$ (反应式(2)). 在该反应中, 将肟的 $\mathrm{N}-\mathrm{OH}$ 转化为更好的离去基团(通过离子化、醚化或者 酯化)十分必要. 也就是说, 醛肜基团氧原子通过活化 剂(活化剂通常是强的亲电试剂如二乙基磷酰氯、氯 磺酸)活化，对于Beckmann裂解反应的发生十分关键 (A表示活化剂丢失卤素或者其他活性基团的部分, $\mathrm{OA}$ 表示离去基团).
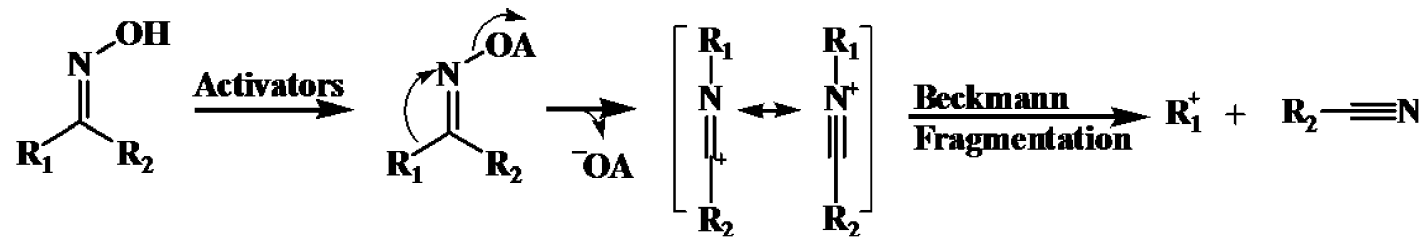

值得一提的是, 如果将 $\mathrm{TCBQ}$ 替换为其他四卤代

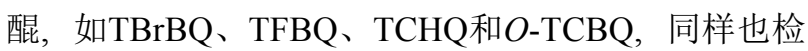
测到了2-CMP的生成，其产率分别为 $97.1 \%$ 、96.8\%、 $74.2 \%$ 和 $100 \%$.

\subsection{2-PAM促进TCBQ加速水解的分子反应机制}

根据上述研究结果以及之前有关Beckmann裂解 反应的相关研究文献，考虑到2-PAM阴离子是一种有 效的 $\alpha$-亲核试剂, 我们提出了2-PAM促进 TCBQ水解的 独特反应机制(图9)：在中性溶液中，2-PAM阴离子 $\left(\mathrm{Py}^{+}\left(\mathrm{CH}_{3}\right)-\mathrm{CH}=\mathrm{N}-\mathrm{O}^{-}\right)$对 $\mathrm{TCBQ}$ 发生亲核进攻, 首先形
成不稳定的过渡态中间产物 $\mathrm{Py}^{+}\left(\mathrm{CH}_{3}\right)-\mathrm{CH}=\mathrm{N}-\mathrm{O}-$ 三氯1,4-苯醌. 该中间体发生第一次Beckmann裂解反应生 成一级水解产物 $\mathrm{TrCBQ}^{-} \mathrm{O}^{-}(2-\mathrm{PAM} / \mathrm{TCBQ}$ 摩尔比例较 低时)和一分子的碳正离子中间体 $\mathrm{Py}^{+}\left(\mathrm{CH}_{3}\right)-\mathrm{C}^{+}=\mathrm{NH}$, 其可共振互变为氮正离子中间体 $\mathrm{Py}^{+}\left(\mathrm{CH}_{3}\right)-\mathrm{C} \equiv \mathrm{NH}^{+}$. 后 者脱除氢质子产生一分子的 $N$-甲基-2-吡啶甲腈(2CMP). 当2-PAM过量时, 生成的 $T r C B Q-\mathrm{O}^{-}$进一步与过 量的2-PAM反应, 通过形成类似的过渡态中间产物, 发 生第二次Beckmann裂解反应生成二羟基化的终产物 DDBQ和另一分子2-CMP.

如果上述提出的两次Beckmann裂解反应机制正 


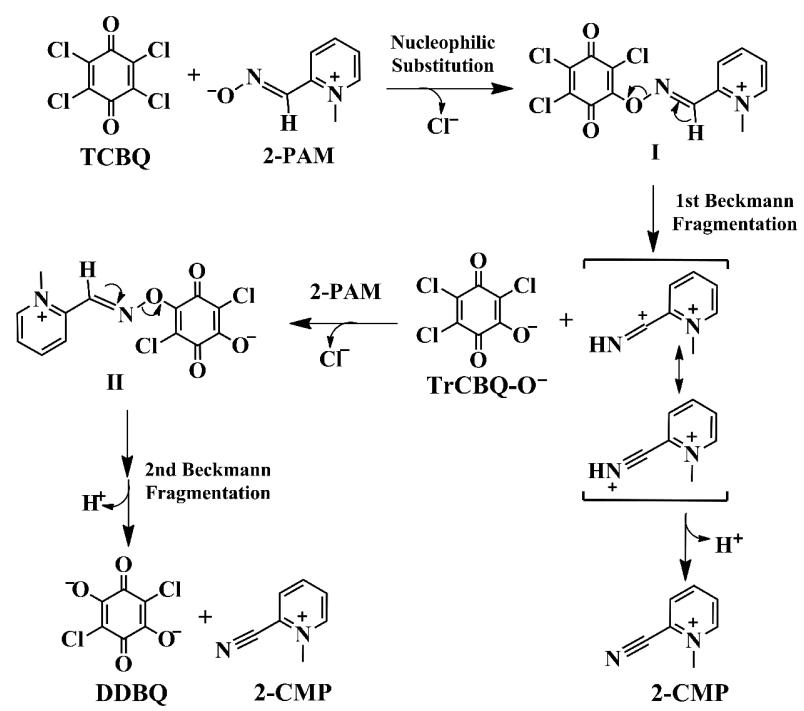

图 92 2-PAM加速 $\mathrm{TCBQ}$ 脱氯水解的可能的分子机制：亲核 进攻伴随着两次非同寻常的Beckmann裂解反应 ${ }^{[61]}$

Figure 9 Proposed mechanism for the dramatic acceleration of dechlorination and dihydroxylation of TCBQ by 2-PAM: nucleophilic attack coupled with an unusually facile double Beckmann fragmentation under physiological conditions [61].

确的话，那么 TCBQ反应后得到的 TrCBQ-OH和DDBQ 中的氧原子应该来源于 2-PAM中的 $\mathrm{N}-\mathrm{OH}$ ，而不是 $\mathrm{H}_{2} \mathrm{O}$. 为了进一步证实该反应的机理, 我们采用同位素 标记的方法, 将2-PAM与 $\mathrm{TCBQ}$ 的反应在 ${ }^{18} \mathrm{O}$ 标记的水 $\left(\left[{ }^{18} \mathrm{O}\right]-\mathrm{H}_{2} \mathrm{O}\right)$ 配制的缓冲溶液中进行。如果水是上述两 种产物中氧原子的来源, 那么在使用 ${ }^{18} \mathrm{O}$ 标记水配制的 反应液中产生的 $\mathrm{TrCBQ}-\mathrm{OH}$ 和DDBQ的分子离子峰, 与未标记的水配制的反应液中获得的产物的分子离子 峰相比，在质荷比 $(\mathrm{m} / \mathrm{z})$ 上应分别显示 2 个或 4 个质量数 的偏移，这样才足以表明 ${ }^{18} \mathrm{O}$ 标记的水参与了反应. 但 是结果发现, 在 ${ }^{18} \mathrm{O}$ 标记水配制的溶液中反应并未观察 到质荷比的位移 ${ }^{[61]}$ ，表明水分子根本没有参与到 TrCBQ-OH和DDBQ产物的形成．这些同位素标记实 验结果进一步支持了所提出的上述反应机制.

\section{5 该Beckmann裂解反应非同寻常的原因}

在Beckmann裂解反应中，酫肜基团中的氧原子活 化对于反应的发生至关重要. 因此, Beckmann裂解反 应通常选择 $O$-活化的醛肟衍生物，或者使醛肜和不同 的活化剂(如浓硫酸、二乙基磷酰氯、氯氟酸等)共存, 通过质子化、醚化或酯化来进行(表 1$)^{[67,68]}$. 据我们所 知，Beckmann裂解反应机制研究发现 TCBQ和其他四 卤代苯醌可作为一类醛肜活化剂，通过形成不稳定的 $O$-三卤代苯醌酫肜中间体，从而自发发生连续两次的 Beckmann裂解反应.

之前报道的Beckmann裂解反应通常是在强酸性 或者加热到特定温度在有机溶剂中发生的一步反 应 $^{[2,67]}$. 2-PAM与 TCBQ的反应是连续两次的Beckmann

表 1 醛肪发生Beckmann裂解的方法 ${ }^{[61]}$

Table 1 The reaction conditions to convert aldoximes to corresponding nitriles [61]

\begin{tabular}{|c|c|c|c|c|c|}
\hline Catalyst & Concentration $(\mathrm{mol} \%)$ & Solvent & Temperature $\left({ }^{\circ} \mathrm{C}\right)$ & Time & Yield (\%) \\
\hline Cyanuryl chloride & 100 & DMF & Room temperature & $4 \mathrm{~h}$ & 100 \\
\hline Propylphosphonic anhydride & 15 & THF & 70 & $1 \sim 3 \mathrm{~h}$ & $>84$ \\
\hline Chloral & 50 & None & $120 \sim 140$ & $2.5 \sim 7.5 \mathrm{~h}$ & $>67$ \\
\hline Chlorosulfonic acid & 3.3 & Toluene & 90 & $0.5 \mathrm{~h}$ & $\approx 100$ \\
\hline Silphos & 20 & THF & Microwave irradiation & $3 \sim 6 \mathrm{~min}$ & $>79$ \\
\hline Silica- $\mathrm{PCl}_{3}$ & - & $\mathrm{MeCN}$ & Room temperature & $8 \sim 10 \mathrm{~h}$ & $>87$ \\
\hline $\mathrm{FeCl}_{3}$-Montmorillonite $\mathrm{K}$ & 40 & Toluene & Reflux & $5 \sim 12 \mathrm{~h}$ & $>91$ \\
\hline Metaboric acid & 3 & None & 140 & $7 \sim 58 \mathrm{~h}$ & $>62$ \\
\hline Diethyl chlorophosphate & 100 & Toluene & Reflux & $20 \sim 120 \mathrm{~h}$ & $\approx 90$ \\
\hline $\mathrm{Ph}_{3} \mathrm{P} / N$-chlorosuccinimide & $120 \sim 150 / 120 \sim 150$ & $\mathrm{CH}_{2} \mathrm{Cl}_{2}$ & Room temperature & $0.5 \mathrm{~h}$ & $75 \sim 97$ \\
\hline$\left(\mathrm{Cl}_{3} \mathrm{CO}\right)_{2} \mathrm{CO}$ & 0.3 & $\mathrm{DMF} / \mathrm{MeCN}$ & Reflux & $1 \mathrm{~h}$ & $>72$ \\
\hline $\mathrm{HCl}$ (gas) & - & - & $70 \sim 130$ & $5 \sim 48 \mathrm{~h}$ & $>67$ \\
\hline $\mathrm{AlCl}_{3} / \mathrm{KI}$ & $100 / 300$ & $\mathrm{H}_{2} \mathrm{O} / \mathrm{MeCN}$ & Room temperature/80 & $0.5 / 6 \mathrm{~h}$ & $70 \sim 96$ \\
\hline
\end{tabular}


裂解反应, 在室温 $\left(25^{\circ} \mathrm{C}\right)$ 、中性(即生理条件), 甚至碱 性条件下均可发生. 这可能是因为我们推测生成的 $O-$ 三氯苯醌醛肜 $(O$-三氯苯醌-2-PAM) 肜醚中间产物能快 速且极易发生裂解反应. 此外, 在最近的研究中发现, 氯代苯醌活化BHA生成的中间体的稳定性与对应的 脱氯羟基化的氯代苯醌共轭酸的 $\mathrm{p} K_{\mathrm{a}}$ 有关 ${ }^{[59]}$. 基于此, 我们推测此规律也适用于中间体 $O$-三氯苯醌醛肟醚. 由于阴离子离去基团的共轭酸 $\operatorname{TrCBQ}-\mathrm{OH}\left(\mathrm{p} K_{\mathrm{a}}: 1.10\right)$ 和DDBQ $\left(\mathrm{p} K_{\mathrm{a} 1}: 0.58 ; \mathrm{p} K_{\mathrm{a} 2}: 2.72\right)$ 的强酸性, 因此 $O$-三氯 代苯醌醛肟醚中间产物的重排反应速率应该非常快. 一旦形成, 这些中间产物就能在室温和中性 $\mathrm{pH}$ 条件下 快速地发生裂解. 这可解释为何 2-PAM在生理学条件 下可以显著加速 $\mathrm{TCBQ}$ (或 $\mathrm{TrCBQ}-\mathrm{OH}$ )水解转化为 DDBQ. 此外, 这解释了为何在本研究中没能分离、鉴 定出推测的反应中间产物, 这一点与大多数 $O$-取代的 肟醚反应明显不同.

\section{$2.62-\mathrm{PAM} / \mathrm{TCBQ} / \mathrm{H}_{2} \mathrm{O}_{2}$ 反应体系的DNA凝胶电} 泳实验

在之前的研究中, 我们发现TCBQ和 $\mathrm{H}_{2} \mathrm{O}_{2}$ 能够通 过不依赖于金属离子的途径产生高活性的羟基自由基 $(\cdot \mathrm{OH})$, 进而导致DNA链断裂 ${ }^{[45,69]}$ 和碱基氧化 ${ }^{[70,71]}$. 上 述研究还发现, 2-PAM能使高毒性的TCBQ水解脱毒 为低毒性的 $T r C B Q-O H$ 和DDBQ, 这意味着2-PAM的 加入可能会抑制TCBQ介导的生物大分子如DNA的氧 化损伤. 为此, 我们考察了2-PAM对TCBQ介导的DNA 氧化损伤的影响. 采用质粒pBR322 DNA作为模型 DNA进行凝胶电泳实验. 环状双链超螺旋pBR322 DNA(form I) 在羟基自由基的作用下会转化为单链断 裂形式(form II)或者双链断裂的线状DNA形式(form III), 不同形式的DNA在电泳条件下移动速率不同, 从 而实现其分离和分析监测, 该方法被用于凝胶电泳实 验以检测DNA链的断裂. 研究结果如预期所示, 2$\mathrm{PAM}$ 可以抑制 $\mathrm{TCBQ} / \mathrm{H}_{2} \mathrm{O}_{2}$ 导致的DNA双链断裂. 为进 一步证实2-PAM可以间接阻止・OH的产生(2-PAM能使 高毒性的 TCBQ 水解脱毒为低毒性的 TrCBQ-OH和 $\mathrm{DDBQ}$, 从而阻止 $\mathrm{TCBQ}$ 与 $\mathrm{H}_{2} \mathrm{O}_{2}$ 产生・ $\mathrm{OH}$ ), 我们还采用 对苯二甲酸(TPA)为捕获剂的苂光方法对此进行检测, 结果发现2-PAM可以有效抑制 $\mathrm{TCBQ} / \mathrm{H}_{2} \mathrm{O}_{2}$ 产生羟基自 由基.

\subsection{2-PAM/TCBQ反应体系的细菌和细胞毒性实验}

为进一步探究2-PAM对 $\mathrm{TCBQ}$ 等卤代醌介导生物 毒性的保护作用, 我们将研究由单独的DNA扩展至细 菌P. phosphoreum 和哺乳动物细胞HepG2两种模式系 统中进行实验. P. phosphoreum 毒性实验, 通常又称为 Microtox 实验 ${ }^{[2]}$, 是一种常见的用来评估化学品、环 境样品、垃圾废弃物处理或修复过程产物的急性水生 毒性的方法. P. phosphoreum 的苂光与其呼吸活动直接 相关, 因此荧光强度可以用于监测化合物对其代谢活 性的影响乃至产生的细胞毒性 ${ }^{[62]}$.

在P. phosphoreum细菌体系中发现, 2-PAM对 $\mathrm{TCBQ}$ 毒性的保护作用非常明显, 并且呈现明显的计 量依赖效应，1.0 mM的2-PAM对TCBQ的保护作用可 达67.2\%. 在对照实验中, 通过比较TCBQ、TrCBQ$\mathrm{OH} 、 \mathrm{DDBQ} 、 2-\mathrm{PAM}$ 和2-CMP对 $P$. phosphoreum细菌 毒性的半抑制浓度 $\left(\mathrm{IC}_{50}\right)$ 发现, DDBQ $\left(\mathrm{IC}_{50}\right.$ 为 $\left.676.9 \mu \mathrm{M}\right)$ 比 TCBQ 和 TrCBQ-OH的毒性要低得多 (TCBQ 和 $\operatorname{TrCBQ}-\mathrm{OH}$ 的 $\mathrm{IC}_{50}$ 分别为 2.6 和 $19.5 \mu \mathrm{M}$ ).

此外, 由于卤代醌和卤代酚可以导致肝毒

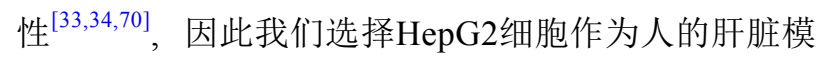
型细胞进行进一步研究. 采用MTT方法来进行 TCBQ 的细胞毒性研究, 结果也表明2-PAM对TCBQ导致的 细胞毒性有保护作用. TCBQ、TrCBQ-OH和DDBQ的 $\mathrm{IC}_{50}$ 值分别为 $2.0 、 94.0$ 和 $613.9 \mu \mathrm{M}$. 有趣的是, 在 $P$. phosphoreum 和 HepG2两种不同细胞实验结果看来, DDBQ的 $\mathrm{IC}_{50}$ 值远远大于 $\mathrm{TCBQ}$, 充分表明在两种细胞 体系中2-PAM将 $\mathrm{TCBQ}$ 转化为 $\mathrm{DDBQ}$ 为解毒过程.

\section{3 可能的环境与生物学意义}

我们发现, 这种新型的两次Beckmann裂解反应机 制并不局限于2-PAM和TCBQ, 而是普遍存在于所有 四卤代醌类化合物与吡啶醛肟类化合物中. 因此, 这 一发现可能具有重要的生物学与环境学意义: 许多广 泛使用的多卤代芳香化合物(公认的POPs和可能的人 体致癌物), 包括多卤代酚类(如普遍存在的 $\mathrm{PCP}$ 与溴代 阻燃剂 $3,3^{\prime}, 5,5^{\prime}$-四溴双酚 $\left.\mathrm{A}\right)$ 、橙剂、六氯苯、六六六 和多氯联苯 $(\mathrm{PCBs})$ 等可通过体内代谢、化学反应或酶 促反应途径脱卤生成相应的卤代醌 ${ }^{[33,34,69,73 \sim 79]}$. 在造纸 厂排放的污水中也检出了多氯代醌类化合物 ${ }^{[80]}$. 最近 有研究报道, 在饮用水消毒副产物和游泳池中检出了 
12 种多卤代醌，其中多种是可疑的膀胱致癌物 ${ }^{[81]}$. 据 了解，与大多数传统的消毒副产物相比，这些多卤代 醌类消毒副产物会导致更强的细胞毒性或者发育毒 性 ${ }^{[47]}$. 细胞研究表明, 卤代醌可以产生活性氧物种 (ROS), 降低细胞谷胱甘肽水平, 影响细胞抗氧化酶, 从而进一步造成细胞氧化应激和细胞DNA、蛋白 质、脂质的氧化损伤 ${ }^{[34,45,47,69]}$. ROS 的形成伴随着 MAPK信号的活化可以解释PCP触发的肿瘤形成机 理 ${ }^{[22]}$. 我们最近的研究发现, 多卤代醌与 $\mathrm{H}_{2} \mathrm{O}_{2}$ 或者有 机氢过氧化物共存可以导致DNA损伤，可将 $5 \mathrm{mdC}$ 氧 化为其甲基氧化产物 ${ }^{[70,83]}$ ，导致脂质过氧化等 ${ }^{[84]}$. 因 此，如何在正常生理条件下破坏或降解这类对哺乳动 物具有潜在致癌性的多卤代醌类物质显得尤为重要.

有趣的是, 4种吡啶盐酫肟类药物(2-PAM、TMB4、Obidoxime、HI-6)均已被应用于临床，以治疗有机 磷农药和神经毒剂的中毒 ${ }^{[1,3]}$. 吡啶盐醛肜类药物之所 以具有良好的解毒效果，是由于吡啶的正电部分能够 很强地结合到酶的活性部位，而醛肜基团则可将有机 磷农药结构中的有机磷部分取代下来. 多卤代醌是一 类致癌中间体，并且最近在饮用水消毒产物中有检出，
正如研究中发现，吡啶醛肜类化合物对卤代醌类化合 物的解毒作用也十分有效. 有趣的是, 研究发现吡啶 盐醛肜类药物可直接与有机磷神经毒剂(如Sarin和Soman, 均为氟代有机磷毒剂)反应, 或者通过活化受抑制 的酶而形成磷酰化朊, 其可在体外分解为磷酸和相应 的腈 ${ }^{[1,85]}$, 该反应机理可能也是通过类似的Beckmann 裂解反应. 值得一提的是, 吡啶盐醛肟类药物也用于 氨基甲酸酯类农药的解毒, 亦可能是通过类似于有机 磷毒剂的胆碱解毒途径 ${ }^{[86,87]}$. 因此, 本研究体系的卤 代醌与2-PAM的亲核取代反应，并同时伴随着发生 Beckmann裂解的反应机制可能是吡啶盐酫肜类药物 的一类共同的但先前未被认知的解毒机制. 近年来, 多 卤代芳香族污染物和吡啶盐醛肜类药物这两类物质不 仅已经引起科研工作者, 而且还引起了社会公众的广 泛关注. 我们的研究结果可能对今后深入研究这两类 与生物医学和环境领域密切相关的化合物具有重要的 化学、生物学和环境学方面的意义. 然而, 尽管研究表 明, 醛肟类药物具有解毒作用, 但其能否作为安全有效 的预防药物，用于预防或治疗多卤代醌类致癌物导致 的肝癌和膀胱癌等疾病尚需进一步研究.

\section{参考文献}

1 Eyer PA, Worek F. Oximes. In: Marrs TC, Maynard RL, Sidell FR, Eds. Chemical Warfare Agents: Toxicology and Treatment. 2nd Ed. Weinheim: Wiley, 2007. 305-329

2 Pereira MMA, Santos PP. Rearrangements of hydroxylamines, oximes and hydroxamic acids. In: Rappoport Z, Liebman JF, Eds. The chemistry of Hydroxylamines, Oximes and Hydroxamic Acids. Weinheim: Wiley, 2009. 386-473

3 Jokanovic M, Prostran M. Curr Med Chem, 2009, 16: 2177-2188

4 Eyer P. Toxicol Rev, 2003, 22: 165-190

5 Noort D, Benschop HP, Black RM. Toxicol Appl Pharmacol, 2002, 184: 116-126

6 Marrs TC. Pharmacol Ther, 1993, 58: 51-66

7 Gray AP. Drug Metab Rev, 1984, 15: 557-589

8 Brown MA, Brix KA. J Appl Toxicol, 1998, 18: 393-408

9 Hackley Jr B, Owens O. J Org Chem, 1959, 24: 1120

10 Green AL, Saville B. J Chem Soc, 1956, 3887

11 Wilson IB. J Am Chem Soc, 1955, 77: 2383-2386

12 Ashani Y, Bhattacharjee AK, Leader H, Saxena A, Doctor BP. Biochem Pharmacol, 2003, 66: 191-202

13 Antonijevic B, Stojiljkovic MP. Clinical Med Res, 2007, 5: 71-82

Eyer P, Hagedorn I, Ladstetter B. Arch Toxicol, 1988, 62: 224-226 
Kalisiak J, Ralph EC, Zhang J, Cashman JR. J Med Chem, 2011, 54: 3319-3330

Dohnal V, Musílek K, Kuča K. J Chromatogr Sci, 2014, 52: 246-251

Dewangan HK, Nagwanshi R, Ghosh KK, Satnami ML. Catal Lett, 2017, 147: 602-611

Mercey G, Verdelet T, Renou J, Kliachyna M, Baati R, Nachon F, Jean L, Renard PY. Acc Chem Res, 2012, 45: 756-766

Ashani Y, Silman I. Hydroxylamines and oximes: Biological properties and potential uses as therapeutic agents. In: Rappoport Z, Liebman JF, Eds. The Chemistry of Hydroxylamines, Oximes and Hydroxamic Acids. Weinheim: Wiley, 2009. 637-646

Zheng W, Yu H, Wang X, Qu W. Environ Int, 2012, 42: 105-116

Ruder AM, Yiin JH. Chemosphere, 2011, 83: 851-861

Demers PA, Davies HW, Friesen MC, Hertzman C, Ostry A, Hershler R, Teschke K. Cancer Causes Control, 2006, 17: 749-758

Guyton KZ, Loomis D, Grosse Y, El Ghissassi F, Bouvard V, Benbrahim-Tallaa L, Guha N, Mattock H, Straif K. Lancet Oncology, 2016, 17: $1637-1638$

Collins JJ, Bodner K, Aylward LL, Wilken M, Swaen G, Budinsky R, Rowlands C, Bodnar CM. J Occup Environ Med, 2009, 51: $1212-1219$

Gunawardana B, Singhal N, Swedlund P. Environ Eng Res, 2011, 16: 187-203

Song RX, Ruan HJ, Jing XY, Liu ZT. Genotoxicity of sodium pentachlorophenol. Carcinog, Teratog Mutag, 2007, 19: 355-357 (in Chinese) [宋瑞霞, 阮鸿洁, 景欣月, 刘征涛. 五氯酚钠的细胞遗传毒性研究. 癌变, 畸变, 突变, 2007, 19: 355-357]

Rao K. Pentachlorophenol: Chemistry, Pharmacology, and Environmental Toxicology. Berlin: Springer Science \& Business Media, 1978

Renner G, Mücke W. Toxicol Environ Chem, 1986, 11: 9-29

Bolton JL, Trush MA, Penning TM, Dryhurst G, Monks TJ. Chem Res Toxicol, 2000, 13: 135-160

Song Y, Wagner BA, Witmer JR, Lehmler HJ, Buettner GR. Proc Natl Acad Sci USA, 2009, 106: 9725-9730

O’Brien PJ. Chem Biol Interact, 1991, 80: 1-41

Halliwell B, Gutteridge JM. Free Radicals in Biology and Medicine. 4th Ed. Oxford: Oxford University Press, 2007

Witte I, Juhl U, Butte W. Mutat Res/DNA Repair Rep, 1985, 145: 71-75

Dahlhaus M, Almstadt E, Henschke P, Lüttgert S, Appel KE. Arch Toxicol, 1996, 70: 457-460

Ehrlich W. Mutat Res Lett, 1990, 244: 299-302

Dahlhaus M, Almstadt E, Appel KE. Toxicol Lett, 1994, 74: 265-274

Wang YJ, Ho YS, Chu SW, Lien HJ, Liu TH, Lin JK. Chem Biol Interact, 1997, 105: 1-16

Jansson K, Jansson V. Mutat Res/Genet Toxicol, 1992, 279: 205-208

Dahlhaus M. Mutat Res/Fundamental Mol Mech Mutagenesis, 1995, 329: 29-36

Lin PH, Nakamura J, Yamaguchi S, La DK, Upton PB, Swenberg JA. Carcinogenesis, 2001, 22: 635-639

Zhu BZ, Kalyanaraman B, Jiang GB. Proc Natl Acad Sci USA, 2007, 104: 17575-17578

Huang R, Wang W, Qian Y, Boyd JM, Zhao Y, Li XF. Anal Chem, 2013, 85: 4520-4529

Li J, Wang W, Moe B, Wang H, Li XF. Chem Res Toxicol, 2015, 28: 306-318

Zhao Y, Anichina J, Lu X, Bull RJ, Krasner SW, Hrudey SE, Li XF. Water Res, 2012, 46: 4351-4360

Qian Y, Wang W, Boyd JM, Wu M, Hrudey SE, Li XF. Environ Sci Technol, 2013, 47: 4426-4433

Li J, Wang W, Zhang H, Le XC, Li XF. Toxicol Sci, 2014, 141: 335-343

Wang W, Qian Y, Jmaiff LK, Krasner SW, Hrudey SE, Li XF. Environ Sci Technol, 2015, 49: 9898-9904

Li J, Moe B, Vemula S, Wang W, Li XF. Environ Sci Technol, 2016, 50: 6744-6752

Wang W, Moe B, Li J, Qian Y, Zheng Q, Li XF. TrAC Trends Anal Chem, 2016, 85: 97-110

Richardson SD, Plewa MJ, Wagner ED, Schoeny R, Demarini DM. Mutat Res/Rev Mutat Res, 2007, 636: 178-242

Villanueva CM, Cantor KP, Grimalt JO, Malats N, Silverman D, Tardon A, Garcia-Closas R, Serra C, Carrato A, Castaño-Vinyals G, Marcos R, Rothman N, Real FX, Dosemeci M, Kogevinas M. Am J Epidemiol, 2007, 165: 148-156

Zhu BZ, Har-El R, Kitrossky N, Chevion M. Free Radic Biol Med, 1998, 24: 360-369

Witte I, Zhu BZ, Lueken A, Magnani D, Stossberg H, Chevion M. Free Radic Biol Med, 2000, 28: 693-700

Zhu BZ, Zhu JG, Mao L, Kalyanaraman B, Shan GQ. Proc Natl Acad Sci USA, 2010, 107: 20686-20690

Shan GQ, Yu A, Zhao CF, Huang CH, Zhu LY, Zhu BZ. J Org Chem, 2015, 80: 180-189

Li F, Huang CH, Xie LN, Qu N, Shao J, Shao B, Zhu BZ. Sci Rep, 2016, 6: 39207 
61 Xie LN, Huang CH, Xu D, Li F, Zhu JG, Shen C, Shao B, Gao HY, Kalyanaraman B, Zhu BZ. J Org Chem, 2017, 82: 13084-13092

62 Sarr DH, Kazunga C, Charles MJ, Pavlovich JG, Aitken MD. Environ Sci Technol, 1995, 29: 2735-2740

63 Lees G, Holmes F, Underhill AE, Powell DB. J Chem Soc A, 1971, 337-341

64 Grob CA. Angew Chem Int Ed, 1969, 8: 535-546

65 Gawley RE. The Beckmann reactions: rearrangements, elimination-additions, fragmentations, and rearrangement-cyclizations. In: Kende AS, Paquette LA, Overman LE, Denmark SE, Eds. Organic Reactions. Vol. 35. Weinheim: Wiley, 1988. 3-53 Drahl MA, Manpadi M, Williams LJ. Angew Chem Int Ed, 2013, 52: 11222-11251

Chandrasekhar S. The Beckmann and related reactions. In: Knochel P, Molander GA, Eds. Comprehensive Organic Synthesis II. Vol. 7. Amsterdam: Elsevier, 2014. 770-800

68 De Luca L, Giacomelli G, Porcheddu A. J Org Chem, 2002, 67: 6272-6274

Zhu BZ, Shan GQ. Chem Res Toxicol, 2009, 22: 969-977

Gupta SS, Stadler M, Noser CA, Ghosh A, Steinhoff B, Lenoir D, Horwitz CP, Schramm KW, Collins TJ. Science, 2002, 296: 326-328

Zhao B, Yang Y, Wang X, Chong Z, Yin R, Song SH, Zhao C, Li C, Huang H, Sun BF, Wu D, Jin KX, Song M, Zhu BZ, Jiang G, Rendtlew Danielsen JM, Xu GL, Yang YG, Wang H. Nucleic Acids Res, 2014, 42: 1593-1605 


\title{
A new detoxification mechanism for aldoxime therapeutic antidotes for chemical warfare nerve-agents
}

\author{
Fu-Rong Ren ${ }^{1,2}$, Xuan Xiao ${ }^{1,2}$, Lin-Na Xie ${ }^{1,2}$, Dan Xu ${ }^{1,2}$, Jie Shao ${ }^{1,2}$, Chun-Hua Huang ${ }^{1,2 *}$, \\ Ben-Zhan Zhu ${ }^{1,2^{*}}$ \\ ${ }^{1}$ State Key Laboratory of Environmental Chemistry and Ecotoxicology, Research Center for Eco-Environmental Sciences, Chinese Academy of \\ Sciences, Beijing 100085, China \\ ${ }^{2}$ University of Chinese Academy of Sciences, Beijing 100049, China \\ *Corresponding author (email: bjsyhch@126.com; bzhu@rcees.ac.cn)
}

\begin{abstract}
Pyridinium aldoximes are best-known as therapeutic antidotes for organophosphorus chemical warfare nerve-agents and pesticides. Polyhalogenated quinones are a class of carcinogenic intermediates and newly identified chlorination disinfection byproducts in drinking water. However, it is not clear what is the exact chemical mechanism underlying such detoxication. Here we showed that pralidoxime, one of the representatives of pyridinium aldoximes, can markedly enhance the dechlorination and hydroxylation of the highly reactive and toxic tetrachloro-1,4-benzoquinone (also called chloranil) in two-consecutive steps to produce the much less toxic 2,5-dichloro-3,6-dihydroxy-1,4benzoquonine (chloranilic acid), with rate acceleration of up to 180000-times. In contrast, no enhancing effect was observed when the hydroxylamine group was blocked via methylation to form $O$-methylated pralidoxime. The major reaction product from pralidoxime was characterized as its corresponding nitrile (2-cyano-1-methylpyridinium chloride). Along with oxygen-18 isotope-labeling studies, we proposed that nucleophilic substitution coupled with an unprecedented double Beckmann fragmentation reaction was responsible for this dramatic enhancement of the detoxification reaction. This paper reviewed the unprecedented double Beckmann fragmentation reaction. Our findings may have broad biological and environmental implications for future research on the aldoxime therapeutic agents and carcinogenic polyhalogenated quinones, which are two important classes of compounds of major biomedical and environmental interest.
\end{abstract}

Keywords: double Beckmann fragmentation, tetrachloro-1,4-benzoquinone ( $p$-chloranil), pyridinium aldoximes, nerveagent antidotes, detoxification

doi: $10.1360 / \mathrm{N} 032018-00084$ 